\title{
A Study on Correlation between Serum Cortisol and Stroke Severity with Serum Cortisol
}

\author{
K.H Jnanendrappa ${ }^{1}$ \\ ${ }^{1}$ MBBS, MD (general medicine), Professor \& HOD, Sri Siddhartha Institute Of Medical Sciences and Research Centre, Tumakuru, Karnataka, India.
}

\section{Abstract}

Background: The stress response that occurs after the event of acute stroke causes the activation of the hypothalamo-pituitary-adrenal (HPA) axis. Certain studies have found that increased serum cortisol level in patients with acute stroke is related to larger infarct volume, greater stroke severity and poor outcome, including death. Subjects and Methods: About 50 new cases of acute ischemic stroke patients, within 72 hours of the acute neurological event, who were admitted in the Sridevi Medical College \& Hospital were included in the study. The study was conducted for a period of 6 months.(June 2018-December 2018). CT Brain was taken during admission to confirm acute ischemic stroke. NIHSS (National Institute of Health Stroke Scale)score for all the patients were assessed for severity at the time of admission. Results: Of the 50 cases, serum cortisol level of 23 cases were within normal limits $(</=690 \mathrm{nmol} / \mathrm{L})$ of which $65.2 \%$ had NIHSS score of less than or equal to 6 and $34.8 \%$ of the cases had NIHSS score more than 6. Conclusion: Among the patients with acute ischemic stroke ,high serum cortisol levels at the time of admission correlates with,1. Clinical severity which is assessed by National Institute of Health Stroke Scale and 2, Poor prognosis and functional outcome after 15 days which is assessed by Modified Rankin Scale.

Keywords: Acute ischemic stroke, HPA axis, serum cortisol, clinical severity, Functional outcome, stroke scales.

Corresponding Author: Dr. K.H Jnanendrappa, MBBS, MD (general medicine), Professor \& HOD, Sri Siddhartha Institute Of Medical Sciences and Research Centre, Tumakuru, Karnataka, India.

Received: December 2019

Accepted: December 2019

\section{Introduction}

A stress response consisting of increased levels of serum cortisol, serum ACTH and catecholamines in the first weeks after acute stroke has been known since the 1950s. The serum cortisol and serum ACTH response has been identified in both cerebral infarction and intracerebral haemorrhage. High s-cortisol levels and s-ACTH have been related to poor outcome. It is, however, not known whether this adrenal glucocorticoid stress response is beneficial or harmful to the damaged brain.

Some researchers suggested that the association between high stress hormone levels and less favorable outcome could be related to cardiac abnormalities resulting from the increased levels of stress-hormones. Whether the stress response is just an epiphenomenon to stroke severity or independently contributes to prognosis remains uncertain. Furthermore, the stress response has not yet been put in perspective by evaluation in the context of parameters generally assumed to be of importance in acute stroke. There are many clinical variables like symptom severity and advanced age which are identified as potential predictors of outcome in patients with acute stroke. But there is a immense need to detect a biomarker for predicting the outcome of acute stroke. The period that ensues after the event of acute stroke can be regarded as a reaction to a stressful event. This stress response causes the activation of the hypothalamo- pituitary-adrenal (HPA) axis and sympathetic nervous system. In acute stroke the first measurable alterations are the endocrine changes because of the alteration in HPA axis. One of the HPA axis- related hormone is cortisol which has a robust circadian rhythm wherein the levels peak typically in the early hours of the day and decline later on.

Cortisol has got a significant effect on the glucose, fat and protein metabolism and cardiovascular reactivity. There are studies which showed that high serum cortisol level associated with very much decreased physical function and impaired level of consciousness . Fiorentino et al showed that salivary cortisol levels can be used as biological marker for identifying patients who are prone for acquiring lower benefits from inpatient rehabilitation services. It is also proved in many studies that, increased cortisol concentrations have been observed in acute ischemic stroke and SAH. Certain studies have found that increased serum and urinary cortisol level in patients with acute stroke is related to larger infarct volume, greater stroke severity and poor outcome, including death. After the acute event, increased serum cortisol level is significantly associated with acute confusional state.

The primary objective of this study dissertation is to test the hypothesis that increased single serum cortisol level is associated with increased severity of acute ischemic stroke. Though cortisol level has diurnal variations it has been 
showed that the normal circadian rhythm of cortisol is suspended during acute stroke and there is no variation of cortisol level in serum throughout the day due to perturbations in the HPA axis.

So this study was designed to study the correlation of serum cortisol on stroke severity.

\section{Subjects and Methods}

1. The aim of the study is to investigate whether serum cortisol and serum ACTH levels are related to stroke severity.

2. To determine whether s-cortisol is related to paraclinical parameters in acute stroke in order to gain knowledge of the relations between s-cortisol and other paraclinical variables of known relevance to stroke.

\section{Source}

This study will be conducted on patients presenting with history of Acute Stroke to Sridevi Hospital.

\section{Study Subjects}

A prospective study will be conducted on 50 patients with history of Acute Stroke presenting within 24 hours admitted to Sridevi Hospital, during the study period between .(June 2018-December 2018).

\section{STUDY PERIOD}

6 months- (June 2018-December 2018).

\section{Inclusion Criteria}

Patients with a history of Acute Stroke presenting within 24 hours will be taken as study subjects.

\section{Exclusion Criteria}

1. Pregnancy

2. Liver disease

3. Age $<18$

4. Patients who are taking following drugs
a. Phenytoin
b. Rifampicin
c. Ketaconazole
d. Steroids

\section{Study Protocol}

A previously designed proforma will be used to collect demographic, stroke particulars, investigations and clinical details of patients.

\section{Results}

Of the 50 cases, serum cortisol level of 23 cases were within normal limits $(</=690 \mathrm{nmol} / \mathrm{L})$ of which $65.2 \%$ had NIHSS score of less than or equal to 6 and $34.8 \%$ of the cases had NIHSS score more than 6 .As the NIHSS score of less than or equal to 6 is considered to be a minor stroke, it is obvious from the above findings that most of the cases with normal cortisol level had no major stroke. Remaining 27 cases had elevated serum cortisol levels. $100 \%$ of the cases with serum cortisol level of more than $690 \mathrm{nmol} / \mathrm{L}$ had NIHSS score above 6.With the $\mathrm{p}$ value of $<0.001$ this is found to be statistically significant.

\section{Discussion}

A total of 50 patients were enrolled in the study who were proven to have Acute Ischemic Stroke by CT Brain which was taken at the time of admission. The minimum age of the patients is 30 years and the maximum age was 88 years. Among the 50 patients, $38 \%$ of the acute ischemic stroke occurred in the age group of 61 to 70 years. And about $57 \%$ were males and $43 \%$ were females. The mean cortisol level was 630.07 in males. The mean cortisol level in females was 658.14 . Of the 50 cases, $26 \%$ were diabetics and $73 \%$ were non diabetics. The mean cortisol level in diabetics was 591.15 7. The mean cortisol level in non diabetics was 660.3840 percent were hypertensives and 60 percent were normotensives. The mean cortisol level in hypertensives was 657.75. The mean cortisol level in normotensives was 632.93 $24 \%$ had CAD and $76 \%$ did not have CAD. The mean cortisol level in cases with CAD was 612.92 the mean cortisol level in non CAD was 652.32.

Of the 50 cases 15 had systolic BP less than $140 \mathrm{~mm} \mathrm{Hg}, 37$ had systolic BP more than or equal to $140 \mathrm{mmHg}$.ie., 26 percent had normal systolic blood pressure and 74 percent had elevated systolic blood pressure. Also, 35 had diastolic $\mathrm{BP}$ less than $90 \mathrm{mmHg}$ and 13 had diastolic BP more than or equal to $90 \mathrm{mmHg}$.ie., 70 per cent had normal diastolic BP and 30 percent had elevated diastolic BP Of the 50 cases, 6 had infarct in the Anterior cerebral artery territory and 42 had infarct in the middle cerebral artery territory and 2 had infarct in the posterior cerebral artery territory.ie., 12 percent had ACA territory infarct, 42 percent had MCA territory infarct, 2 percent had PCA territory infarct. It is clear that majority of the cases had MCA territory infarct Of the 50 cases, serum cortisol level of 23 cases were within normal limits $(</=690 \mathrm{nmol} / \mathrm{L})$ of which $65.2 \%$ had NIHSS score of less than or equal to 6 and $34.8 \%$ of the cases had NIHSS score more than 6 .As the NIHSS score of less than or equal to 6 is considered to be a minor stroke, it is obvious from the above findings that most of the cases with normal cortisol level had no major stroke.

Remaining 27 cases had elevated serum cortisol levels.100\% of the cases with serum cortisol level of more than 690nmol/L had NIHSS score above 6. With the $\mathrm{p}$ value of $<0.001$ this is found to be statistically significant. As the NIHSS score above 6 is considered to be moderate to severe stroke, it is obvious from the above observation that nearly all cases with elevated cortisol level had moderate to severe stroke.

Of the 50 cases, serum cortisol levels of 23 cases were within normal limits $(/=690 \mathrm{nmol} / \mathrm{L})$ of which $78.3 \%$ had MRS score less than or equal to 3 and $21.7 \%$ had MRS score more than 3.Since MRS score is a measure of functional outcome and any score less than or equal to 3 is considered to have a favourable outcome, it is clear from the above findings that most of the cases with normal serum cortisol had a favourable outcome with minimal neurological impairment. And in the remaining 27 cases which had serum cortisol level more than $690 \mathrm{nmol} / \mathrm{L}, 3.7 \%$ had MRS score of less than or equal to 3 and $96.3 \%$ had MRS score of more than 3 .With the $\mathrm{p}$ value of $<0.001$, this is statistically significant .Since MRS score more than 3 is associated with bad 
outcome, most of the cases with elevated serum cortisol had a poor outcome with severe neurological impairment.

Also among the 50 cases, 5 cases showed mortality who had elevated serum cortisol $(>690 \mathrm{nmol} / \mathrm{L})$ at the time of admission.

\section{Conclusion}

Among the patients with acute ischemic stroke, high serum cortisol levels at the time of admission correlates with,

1. Clinical severity which is assessed by National Institute of Health Stroke Scale and

2. Poor prognosis and functional outcome after 15 days which is assessed by Modified Rankin Scale.

\section{References}

1. Otte C, Hart S, Neylan TC, Marmar CR, Yaffe K, Mohr DC. A metaanalysis of cortisol response to challenge in human aging: importance of gender. Psychoneuroendocrinology. 2005;30:80-91

2. MacLullich A, Deary IJ, Starr JM, Ferguson KJ, Wardlaw JM, Seckl JR. Plasma cortisol levels, brain volumes and cognition in healthy elderly men. Psychoneuroendocrinology. 2005;30(5):505-515.
3. Miller AH, Spencer RL, McEwen BS, Stein M. Depression, adrenal steroids and the immune system. Ann Intern Med. 1993;25(5):481487.

4. Mitchell A. Clinical implications of poststroke hypothalamo-pituitary adrenal axis dysfunction: a critical literature review. J Stroke Cerebrovascular Dis. 1997;6(6):377-388.

5. Brown E, Woolston D, Frol A, Bobadilla L, Khan DA, Hanczyc M, Rush AJ, Fleckenstein J, Babcock E, Cullum CM. Hippocampal volume, spectroscopy, cognition, and mood in patients receiving corticosteroid therapy. Biol Psychiatry. 2004;55:538-545.

6. van Munster B, Bisschop PH, Zwinderman AH, Korevaar JC, Endert E, Wiersinga WJ, van Oosten HE, Goslings JC, de Rooij SE. Cortisol, interleukins and S100B in delirium in the elderly. Brain Cogn. 2010;74:18-23.

7. Pearson A, de Vries A, Middleton SD, Gillies F, White TO, Armstrong IR, Andrew R, Seckl JR, MacLullich AM. Cerebrospinal fluid cortisol levels are higher in patients with delirium versus controls. BMC Res Notes. 2010;3:33.

8. Lee B, Thomas AG, McAtee MJ. Associations of salivary cortisol with cognitive function in the Baltimore memory study. Arch Gen Psychiatry. 2007;64:810-818.

9. Kumari M, et al. Association of diurnal patterns in salivary cortisol with all-cause and cardiovascular mortality: findings from the Whitehall II study. J Clin Endocrinol Metab. 2011;96(5):1478-1485.

10. 10. Franceschini R, et al. Endocrine abnormalities and outcome of ischaemic stroke. Biomed Pharmacother. 2001;55(8):458-465.

Copyright: () the author(s), 2019. It is an open-access article distributed under the terms of the Creative Commons Attribution License (CC BY 4.0), which permits authors to retain ownership of the copyright for their content, and allow anyone to download, reuse, reprint, modify, distribute and/or copy the content as long as the original authors and source are cited.

How to cite this article: Jnanendrappa KH. A Study on Correlation between Serum Cortisol and Stroke Severity with Serum Cortisol. Acad. J Med. 2019;2(2):179-81.

DOI: dx.doi.org/10.21276/ajm.2019.2.2.46 